\title{
ON THE $T^{3}$-GOWDY SYMMETRIC EINSTEIN-MAXWELL EQUATIONS
}

\author{
HANS RINGSTRÖM
}

\begin{abstract}
Recently, progress has been made in the analysis of the expanding direction of Gowdy spacetimes. The purpose of the present paper is to point out that some of the techniques used in the analysis can be applied to other problems. The essential equations in the case of the Gowdy spacetimes can be considered as a special case of a wider class of variational problems. Here we are interested in the asymptotic behaviour of solutions to this class of equations. Two particular members arise when considering the $T^{3}$-Gowdy symmetric Einstein-Maxwell equations and when considering $T^{3}$-Gowdy symmetric IIB superstring cosmology. The main result concerns the rate of decay of a naturally defined energy. A subclass of the variational problems can be interpreted as wave map equations, and in that case one gets the following picture. The non-linear wave equations one ends up with have as a domain the positive real line in cartesian product with the circle. For each point in time, the wave map can thus be seen as a loop in some Riemannian manifold. As a consequence of the decay of the energy mentioned above, the length of the loop converges to zero at a specific rate.
\end{abstract}

\section{INTRODUCTION}

1.1. Background. In the study of the expanding direction of cosmological models, the results can roughly be divided into two groups; small data results and results obtained for cases with symmetry. This is due to the fact that analyzing Einstein's equations in general seems to be out of reach at this time. In this paper, we shall be concerned with a situation in which there is symmetry, but we shall consider general data within the given symmetry class. In [2], Gowdy introduced a class of vacuum spacetimes with a two dimensional group of isometries, see also [1], and in [3] the fundamental global existence result was proved. The symmetry is consistent with the following topologies on the spatial Cauchy surfaces: $T^{3}, S^{2} \times S^{1}, S^{3}$ and the Lens spaces. Since one only expects there to be an expanding direction in the case of $T^{3}$-topology, we shall only be concerned with this case here. In the vacuum case, the essence of the equations is contained in a wave map problem, where the target is the hyperbolic plane. If one considers the Einstein-Maxwell equations under the same symmetry conditions, one obtains a similar variational problem, [5], but, at least in the form given in [5], it is not a wave map problem. Finally, when considering IIB superstring cosmology under the above sort of symmetry condition, one ends up with a wave map problem similar to the vacuum case, the only difference being the target, see [4]. Recently, progress has been made concerning the asymptotic behaviour of Gowdy spacetimes in the expanding direction, see [6], i.e. in the special case that one has a wave map problem with the hyperbolic space as a target. In fact, it turned out to be possible to carry out quite a detailed analysis of the 
corresponding wave map equations. The question then arises if the analysis can be generalized to other targets, and if there is a relation between the geometry of the target and the type of results obtained in the Gowdy case. Furthermore, it is of interest to analyze the Einstein-Maxwell case, in which the problem, at least as it is formulated in [5], is not in wave map form. The analysis in [6] consists of several steps, the first one being that of obtaining decay for the energy. For each point in time, the wave map can be viewed as a loop in the target space, and the fact that the energy decays implies that the length of the loop with respect to the metric on the target space converges to zero at a specific rate. The second step consists of a detailed analysis of the behaviour of the solution, but this depends strongly on the symmetries of the target, so it can not be expected to be generalized very easily. That is not to say that nothing can be done, but in this paper we are only going to consider the decay of the energy. The focus of the current paper is on the resulting variational problem, and we shall not consider the consequences of the conclusions obtained in this paper on the space time geometry. In fact the consequences for the corresponding spacetimes are not particularly far reaching. What is done here should rather be understood as a first step in analyzing the asymptotics.

1.2. Generalities. We will throughout consider the Euler-Lagrange equations corresponding to a Lagrangian density of the form

$$
\mathcal{L}=\mathcal{L}_{1}+\mathcal{L}_{2}
$$

where

and

$$
\mathcal{L}_{1}=\frac{t}{2} \bar{g}_{\alpha \beta}(f)\left\{-f_{t}^{\alpha} f_{t}^{\beta}+f_{\theta}^{\alpha} f_{\theta}^{\beta}\right\}
$$

$$
\mathcal{L}_{2}=\frac{1}{2} \bar{h}_{\gamma \delta}(f)\left\{-g_{t}^{\gamma} g_{t}^{\delta}+g_{\theta}^{\gamma} g_{\theta}^{\delta}\right\}
$$

Here $\theta \in S^{1}, t \in \mathbb{R}_{+}=(0, \infty), \bar{g}_{\alpha \beta}$ defines a smooth map from $\mathbb{R}^{n_{1}}$ to the symmetric positive definite $n_{1} \times n_{1}$ matrices and $\bar{h}_{\gamma \delta}$ defines a smooth map from $\mathbb{R}^{n_{1}}$ to the symmetric positive definite $n_{2} \times n_{2}$ matrices. If $\mathcal{L}_{2}=0$, the arguments below all go through; one only has to set all terms involving $\bar{h}$ to zero. In that case, the relevant equations are those of a wave map problem. In fact, consider a map from $\mathbb{R}_{+} \times T^{2}$ with the metric

$$
g_{0}=-d t^{2}+d \theta^{2}+t^{2} d \phi^{2}
$$

to $\mathbb{R}^{n_{1}}$ with some metric $\bar{g}$. Then the wave map equations corresponding to such a map which is independent of the $\phi$-variable are the same as the Euler-Lagrange equations corresponding to the Lagrangian density $\mathcal{L}_{1}$ above.

Assume from now on that $f: \mathbb{R}_{+} \times S^{1} \rightarrow \mathbb{R}^{n_{1}}$ and $g: \mathbb{R}_{+} \times S^{1} \rightarrow \mathbb{R}^{n_{2}}$ satisfy the Euler-Lagrange equations corresponding to $\mathcal{L}$. Define $H$ and $\hat{H}$ by

$$
t H=\hat{H}=\hat{H}_{1}+\hat{H}_{2}
$$

where $H_{i}$ and $\hat{H}_{i}, i=1,2$, are defined by

$$
t H_{1}=\hat{H}_{1}=\frac{t}{2} \int_{S^{1}} \bar{g}_{\alpha \beta}\left\{f_{t}^{\alpha} f_{t}^{\beta}+f_{\theta}^{\alpha} f_{\theta}^{\beta}\right\} d \theta, \quad t H_{2}=\hat{H}_{2}=\frac{1}{2} \int_{S^{1}} \bar{h}_{\alpha \beta}\left\{g_{t}^{\gamma} g_{t}^{\delta}+g_{\theta}^{\gamma} g_{\theta}^{\delta}\right\} d \theta
$$

Then

$$
\frac{d \hat{H}}{d t}=\frac{1}{t} \int_{S^{1}} \mathcal{L}_{1} d \theta \quad \text { and } \quad \frac{d H}{d t}=-\frac{1}{t} \int_{S^{1}} \bar{g}_{\alpha \beta} f_{t}^{\alpha} f_{t}^{\beta} d \theta-\frac{1}{t} H_{2}
$$


Note that as a consequence, $H$ is monotonically decaying, but that it is not apriori clear that it tends to zero as $t$ tends to infinity. Another important consequence of the geometric setting is the following. Let

$$
\begin{aligned}
\mathcal{A} & =\frac{t}{2} \bar{g}_{\alpha \beta}\left(f_{t}^{\alpha}+f_{\theta}^{\alpha}\right)\left(f_{t}^{\beta}+f_{\theta}^{\beta}\right)+\frac{1}{2} \bar{h}_{\gamma \delta}\left(g_{t}^{\gamma}+g_{\theta}^{\gamma}\right)\left(g_{t}^{\delta}+g_{\theta}^{\delta}\right), \\
\mathcal{B} & =\frac{t}{2} \bar{g}_{\alpha \beta}\left(f_{t}^{\alpha}-f_{\theta}^{\alpha}\right)\left(f_{t}^{\beta}-f_{\theta}^{\beta}\right)+\frac{1}{2} \bar{h}_{\gamma \delta}\left(g_{t}^{\gamma}-g_{\theta}^{\gamma}\right)\left(g_{t}^{\delta}-g_{\theta}^{\delta}\right) .
\end{aligned}
$$

Then

$$
\left(\partial_{t}-\partial_{\theta}\right) \mathcal{A}=\left(\partial_{t}+\partial_{\theta}\right) \mathcal{B}=\frac{1}{t} \mathcal{L}_{1}
$$

Note that (4) is in fact a consequence of (7). Finally, it will be convenient to introduce the notation

$$
\mathcal{K}_{1}=\bar{g}_{\alpha \beta} f_{t}^{\alpha} f_{t}^{\beta}, \quad \mathcal{P}_{1}=\bar{g}_{\alpha \beta} f_{\theta}^{\alpha} f_{\theta}^{\beta},
$$

and

$$
H_{1, K}=\frac{1}{2} \int_{S^{1}} \mathcal{K}_{1} d \theta, \quad H_{1, P}=\frac{1}{2} \int_{S^{1}} \mathcal{P}_{1} d \theta
$$

1.3. Metrics. We shall consider metrics of the form

$$
\begin{aligned}
(8) \bar{g}= & \sum_{i=1}^{l} d P^{i} \otimes d P^{i}+\sum_{j=1}^{m} \mathcal{Q}^{j} d Q^{j} \otimes d Q^{j} \\
& +\sum_{k=1}^{n} \mathcal{R}^{k}\left[d R^{k}+\sum_{j_{1}, j_{2}=1}^{m} \alpha_{j_{1} j_{2}}^{k} Q^{j_{1}} d Q^{j_{2}}\right] \otimes\left[d R^{k}+\sum_{j_{1}, j_{2}=1}^{m} \alpha_{j_{1} j_{2}}^{k} Q^{j_{1}} d Q^{j_{2}}\right],
\end{aligned}
$$

where

$$
\mathcal{Q}^{j}=\exp \left[\sum_{i=1}^{l} \alpha_{i}^{j} P^{i}\right], \quad \mathcal{R}^{k}=\exp \left[\sum_{i=1}^{l} \beta_{i}^{k} P^{i}\right],
$$

where $\alpha_{i}^{j}, \beta_{i}^{k}$ and $\alpha_{j_{1} j_{2}}^{k}$ are constants. Most arguments will require these constants to satisfy some algebraic relations which we now define.

Definition 1.1. Consider a metric of the form (8). We shall say that it scales if $\alpha_{j_{1} j_{2}}^{k} \neq 0$ implies

$$
\mathcal{R}^{k}=\mathcal{Q}^{j_{1}} \mathcal{Q}^{j_{2}} .
$$

Furthermore, we shall say that the metric is ordered if $\alpha_{j_{1} j_{2}}^{k} \neq 0$ for some $k, j_{1}$ and $j_{2}$ implies that $\alpha_{j_{2}^{\prime} j_{1}}^{k^{\prime}}=0$ for all $k^{\prime}$ and $j_{2}^{\prime}$.

Remark. Note that if $n=0$, then the metric scales and is ordered. The motivation for the terminology scales is as follows. Define a map by

$$
P^{i} \rightarrow P^{i}+\Delta^{i}, \quad Q^{j} \rightarrow Q^{j} \exp \left[-\frac{1}{2} \sum_{i=1}^{l} \alpha_{i}^{j} \Delta^{i}\right], \quad R^{k} \rightarrow R^{k} \exp \left[-\frac{1}{2} \sum_{i=1}^{l} \beta_{i}^{k} \Delta^{i}\right],
$$

where $\Delta^{i} \in \mathbb{R}, i=1, \ldots, l$. This map is an isometry of (8) if the metric scales.

We shall also assume that the metric $\bar{h}$ has a special form. 
Definition 1.2. Let $\bar{g}$ be a metric of the form (8) and let $n_{1}=l+m+n$. A smooth map $\bar{h}$ from $\mathbb{R}^{n_{1}}$ to the set of symmetric positive definite $n_{2} \times n_{2}$ matrices will be called a $\bar{g}$-metric if $\bar{h}$ only depends on $(P, Q) \in \mathbb{R}^{l+m}$ and if there are constants $C_{1}$ and $C_{2}$ such that

$$
\begin{aligned}
\left|\frac{\partial \bar{h}_{\gamma \delta}}{\partial P^{i}}(P, Q) V^{\gamma} W^{\delta}\right| & \leq C_{1} \bar{h}_{\gamma \delta}(P, Q)\left(V^{\gamma} V^{\delta}+W^{\gamma} W^{\delta}\right), \\
\left|\left(\mathcal{Q}^{j}\right)^{-1 / 2} \frac{\partial \bar{h}_{\gamma \delta}}{\partial Q^{j}}(P, Q) V^{\gamma} W^{\delta}\right| & \leq C_{2} \bar{h}_{\gamma \delta}(P, Q)\left(V^{\gamma} V^{\delta}+W^{\gamma} W^{\delta}\right)
\end{aligned}
$$

for arbitrary $(P, Q) \in \mathbb{R}^{l+m}, V, W \in \mathbb{R}^{n_{2}}$ and $i, j$. (In the second inequality, there is no summation on $j$ ).

When considering Einstein's vacuum equations with Gowdy $T^{3}$-symmetry, one obtains a problem of the form above, with $\mathcal{L}_{2}=0$ and $\bar{g}$ given by

$$
g_{H}=d P^{2}+e^{2 P} d Q^{2} .
$$

The corresponding Riemannian manifold is in fact isometric to hyperbolic space. In [4], Makoto Narita considered $T^{3}$-Gowdy symmetric IIB superstring cosmology, and the relevant problem that arises in this case is of the above type, with $\mathcal{L}_{2}=0$ and $\bar{g}$ defined by $g_{H}+g_{H S}$, where

$$
g_{H S}=d \phi^{2}+d \beta^{2}+e^{\sqrt{3} \beta+\phi} d \chi^{2}+e^{-\sqrt{3} \beta+\phi} d \sigma_{2}^{2}+e^{2 \phi}\left(d \sigma_{1}-\chi d \sigma_{2}\right)^{2} .
$$

Note that in this case, the equations for $(Q, P)$ and the equations for $\left(\phi, \chi, \beta, \sigma_{1}, \sigma_{2}\right)$ decouple, so that the relevant metric to consider is $g_{H S}$. Furthermore, the metrics $g_{H}$ and $g_{H S}$ scale and are ordered. Note also that $m+1$-dimensional hyperbolic space falls into the above category with $\mathcal{L}_{2}=0$. However, the corresponding wave map problem has no physical interpretation as far as we are aware. Finally, the Einstein-Maxwell-Dilaton-Axion system considered in [5] is the Euler-Lagrange equations corresponding to $\mathcal{L}$, with

$$
\bar{g}=d Z^{2}+4 d \phi^{2}+e^{-2 Z} d X^{2}+e^{4 a_{A} \phi} d \kappa^{2}
$$

and

$$
\bar{h}=4 e^{-2 a_{M} \phi}\left[e^{Z} d \omega^{2}+e^{-Z}(d \chi-X d \omega)^{2}\right] .
$$

By the change of variables $\psi=2 \phi$, the metric $\bar{g}$ has the form (8). Furthermore $\bar{h}$ is a $\bar{g}$-metric. Note that the equations (14) and (15) in [5] contain a mistake; expressions of the form

$$
\dot{X}(X \dot{\omega}-\dot{\chi})+X^{\prime}\left(X \omega^{\prime}-\chi^{\prime}\right)
$$

appear where expressions of the form

$$
\dot{X}(X \dot{\omega}-\dot{\chi})-X^{\prime}\left(X \omega^{\prime}-\chi^{\prime}\right)
$$

should appear. The Einstein-Maxwell equations are a special case of the above, when one puts $\kappa=\phi=0$ and lets the constants $a_{A}=a_{M}=0$. Note also that one can put the Maxwell part to zero. The equations for the Dilaton-Axion then decouple and has a form very similar to that of Gowdy. Analyzing the asymptotics in this case should for this reason be very similar to the analysis of Gowdy presented in $[6]$. 
1.4. Equations. Let $\bar{g}$ be of the form (8), let $\bar{h}$ be a $\bar{g}$-metric and let the corresponding Lagrangian density be defined by (1). Before writing down the equations, let us define

$$
A^{k}=R_{t}^{k}+\sum_{j_{1}, j_{2}=1}^{m} \alpha_{j_{1} j_{2}}^{k} Q^{j_{1}} Q_{t}^{j_{2}} \quad \text { and } \quad B^{k}=R_{\theta}^{k}+\sum_{j_{1}, j_{2}=1}^{m} \alpha_{j_{1} j_{2}}^{k} Q^{j_{1}} Q_{\theta}^{j_{2}}
$$

The equations come in blocks. Let us start with the equations that come from varying $R^{k}$. We get

$$
\partial_{t}\left(t \mathcal{R}^{k} A^{k}\right)-\partial_{\theta}\left(t \mathcal{R}^{k} B^{k}\right)=0
$$

For the $P^{i}$ :s, we have

$$
\begin{aligned}
\partial_{t}\left(t P_{t}^{i}\right)-\partial_{\theta}\left(t P_{\theta}^{i}\right)= & \frac{t}{2} \sum_{j=1}^{m} \alpha_{i}^{j} \mathcal{Q}^{j}\left[\left(Q_{t}^{j}\right)^{2}-\left(Q_{\theta}^{j}\right)^{2}\right]+\frac{t}{2} \sum_{k=1}^{n} \beta_{i}^{k} \mathcal{R}^{k}\left[\left(A^{k}\right)^{2}-\left(B^{k}\right)^{2}\right] \\
& +\frac{1}{2} \frac{\partial \bar{h}_{\gamma \delta}}{\partial P^{i}}\left(g_{t}^{\gamma} g_{t}^{\delta}-g_{\theta}^{\gamma} g_{\theta}^{\delta}\right)
\end{aligned}
$$

For the $Q^{j}: \mathrm{s}$, we have

$$
\begin{aligned}
\partial_{t}\left(t \mathcal{Q}^{j} Q_{t}^{j}\right)-\partial_{\theta}\left(t \mathcal{Q}^{j} Q_{\theta}^{j}\right) & +t \sum_{k=1}^{n} \mathcal{R}^{k} \sum_{o=1}^{m}\left(\alpha_{o j}^{k}-\alpha_{j o}^{k}\right)\left(Q_{t}^{o} A^{k}-Q_{\theta}^{o} B^{k}\right) \\
& =\frac{1}{2} \frac{\partial \bar{h}_{\gamma \delta}}{\partial Q^{j}}\left(g_{t}^{\gamma} g_{t}^{\delta}-g_{\theta}^{\gamma} g_{\theta}^{\delta}\right)
\end{aligned}
$$

where we have used (12). Note that second derivatives of $Q$ appear in (12), but that these can be eliminated using (14). Finally, for the $g^{\gamma}$, we have

$$
\partial_{t}\left(\bar{h}_{\gamma \delta} g_{t}^{\delta}\right)=\partial_{\theta}\left(\bar{h}_{\gamma \delta} g_{\theta}^{\delta}\right) \text {. }
$$

1.5. Results. In section 2, we prove that given smooth initial data for (12)-(15) at some $t_{0} \in \mathbb{R}_{+}$, there is a unique smooth solution defined for all $t \in \mathbb{R}_{+}$. The argument concerning the decay of the energy is in a natural way divided into two parts. First we prove that we get the desired decay if the energy is small enough to start with, and then we prove that the energy always decays to zero. Since the conditions for the small data result are weaker than for the large data result, we write it down here.

Proposition 1. Fix a metric $\bar{g}$ of the form (8), which scales, and a $\bar{g}$-metric $\bar{h}$. Then there is an $\epsilon>0$ such that if $H\left(t_{0}\right) \leq \epsilon$ for a solution to (12)-(15), where $t_{0} \in \mathbb{R}_{+}$and $H$ is defined in (3), there is a $T$ and a $C$ such that

$$
H(t) \leq \frac{C}{t}
$$

for all $t \geq T$.

Note that in [6], it was proved that if $\mathcal{L}_{2}=0$ and $\bar{g}=g_{H}$, then

$$
\left|t H(t)-c_{H}\right| \leq \frac{C}{t}
$$

and that $c_{H}=0$ if and only if the solution is independent of $\theta$. Note that $t^{2} H(t)$ is constant for solutions that are independent of $\theta$. Proving the opposite implication is however more difficult. The decay obtained in Proposition 1 can in other words 
not be improved in general. In the above example, $H=H_{1}$, and so one could of course hope that $\mathrm{H}_{2}$ satisfies a better decay estimate. The following, rather uninteresting, example shows that this in not the case in general. Let $\bar{h}$ be the standard Riemannian metric on $\mathbb{R}$. Then $g^{1}$ satisfies the standard wave equation on the cylinder. Since $g^{1}(t, \theta)=t$ is a solution of this equation, we cannot improve the estimate for $\mathrm{H}_{2}$ in general. For large data, we also need to require that the metric be ordered.

Theorem 1. Consider a solution to (12)-(15) corresponding to a metric $\bar{g}$ of the form (8), which scales and is ordered, and $a \bar{g}$-metric $\bar{h}$. Let $H$ be defined by (3). Then there is a $C$ and a $T$ such that

$$
H(t) \leq \frac{C}{t}
$$

for all $t \geq T$.

Let us consider the case $\mathcal{L}_{2}=0$. Then for a fixed $t$, the solution defines a loop in the Riemannian manifold $\left(\mathbb{R}^{l+m+n}, \bar{g}\right)$, and the length of the loop with respect to this Riemannian metric is given by

$$
\int_{S^{1}}\left(\bar{g}_{\alpha \beta} f_{\theta}^{\alpha} f_{\theta}^{\beta}\right)^{1 / 2} d \theta \leq(2 \pi)^{1 / 2}\left(\int_{S^{1}} \bar{g}_{\alpha \beta} f_{\theta}^{\alpha} f_{\theta}^{\beta} d \theta\right)^{1 / 2} \leq 2 \pi^{1 / 2} H^{1 / 2} \leq C t^{-1 / 2}
$$

\section{Global existence}

The arguments in this section are of course standard, but we wish to prove the following for the sake of completeness.

Theorem 2. Consider the Euler-Lagrange equations corresponding to $\mathcal{L}$ defined in (1), where $\bar{g}$ is of the form (8) and $\bar{h}$ is any smooth mapping from $\mathbb{R}^{l+m+n}$ to the symmetric positive definite $n_{2} \times n_{2}$ matrices for some $n_{2} \in \mathbb{N}$. Given smooth initial data given at some $t_{0} \in \mathbb{R}_{+}$, there is a unique smooth solution to these equations on all of $\mathbb{R}_{+}$.

Proof. Let $\mathcal{A}$ and $\mathcal{B}$ be defined by (5) and (6) and let

$$
F_{1}(u, \theta)=\mathcal{A}(u, \theta-u), \quad F_{2}(u, \theta)=\mathcal{B}(u, \theta+u), \quad E_{i}(u)=\sup _{\theta \in S^{1}} F_{i}(u, \theta)
$$

and

$$
E=E_{1}+E_{2}
$$

By (7), we have

$$
\begin{aligned}
\left|F_{1}\left(u_{1}, \theta\right)-F_{1}\left(u_{0}, \theta\right)\right| & =\left|\int_{u_{0}}^{u_{1}} \partial_{u} F_{1}(u, \theta) d u\right|=\left|\int_{u_{0}}^{u_{1}} \frac{1}{u} \mathcal{L}_{1}(u, \theta-u) d u\right| \\
& \leq\left|\int_{u_{0}}^{u_{1}} \frac{1}{2 u} E(u) d u\right|
\end{aligned}
$$

and similarly for $F_{2}$. Taking supremum over $\theta$ and then adding, we get

$$
E\left(u_{1}\right) \leq E\left(u_{0}\right)+\left|\int_{u_{0}}^{u_{1}} \frac{1}{u} E(u) d u\right| .
$$


For $u_{1} \geq u_{0}$, we can apply Grönwall's lemma to obtain

$$
E\left(u_{1}\right) \leq \frac{u_{1}}{u_{0}} E\left(u_{0}\right)
$$

for all $u_{1} \geq u_{0}$. In order to analyze the case $u_{1} \leq u_{0}$, define

$$
h(u)=E\left(u_{0}\right)-\int_{u_{0}}^{u} \frac{1}{v} E(v) d v .
$$

Then

$$
h^{\prime}=-\frac{1}{u} E \geq-\frac{1}{u} h
$$

This implies

$$
E\left(u_{1}\right) \leq \frac{u_{0}}{u_{1}} E\left(u_{0}\right)
$$

for all $u_{1} \leq u_{0}$. Thus $E$ is bounded on compact subintervals of $\mathbb{R}_{+}$. Consequently, $P$ is bounded on such intervals, so that $\mathcal{Q}^{j}$ and $\mathcal{R}^{k}$ are bounded on compact subintervals of $\mathbb{R}_{+}$. Consequently, the sup norm of $P$ and $Q$ and the first derivatives of $P$ and $Q$ are bounded on compact subintervals of $\mathbb{R}_{+}$. Due to the form of the metric, this can then be used to get control of $R$ and its first derivatives. Finally, we get control of the $g^{\gamma}$ since we have control over $\bar{h}_{\gamma \delta}(P, Q, R)$. Using this together with energy estimates, one can control the higher order derivatives in $L^{2}$, and thus one obtains global existence.

\section{SMAll DATA}

For the purposes of this section, we fix a metric $\bar{g}$ of the form (8), which scales, and a $\bar{g}$-metric $\bar{h}$. Consider a solution to (12)-(15). Let us first note some facts concerning the spatial variation of different objects. We shall use the notation

$$
\left\langle P^{i}\right\rangle=\frac{1}{2 \pi} \int_{S^{1}} P^{i} d \theta .
$$

and

$$
\overline{\mathcal{Q}}^{j}=\exp \left[\sum_{i=1}^{l} \alpha_{i}^{j}\left\langle P^{i}\right\rangle\right], \quad \overline{\mathcal{R}}^{k}=\exp \left[\sum_{i=1}^{l} \beta_{i}^{k}\left\langle P^{i}\right\rangle\right]
$$

We shall also use the notation

$$
\bar{A}^{k}=R_{t}^{k}+\sum_{j_{1}, j_{2}=1}^{m} \alpha_{j_{1} j_{2}}^{k}\left\langle Q^{j_{1}}\right\rangle Q_{t}^{j_{2}}, \quad \bar{B}^{k}=R_{\theta}^{k}+\sum_{j_{1}, j_{2}=1}^{m} \alpha_{j_{1} j_{2}}^{k}\left\langle Q^{j_{1}}\right\rangle Q_{\theta}^{j_{2}}
$$

and

$$
\hat{B}^{k}=R^{k}-\left\langle R^{k}\right\rangle+\sum_{j_{1}, j_{2}=1}^{m} \alpha_{j_{1} j_{2}}^{k}\left\langle Q^{j_{1}}\right\rangle\left(Q^{j_{2}}-\left\langle Q^{j_{2}}\right\rangle\right) .
$$


Lemma 1. Consider a solution to (12)-(15). Then for $t \geq t_{0}>0$,

$$
\begin{aligned}
\left\|P^{i}-\left\langle P^{i}\right\rangle\right\|_{C^{0}\left(S^{1}, \mathbb{R}\right)} & \leq C H_{1}^{1 / 2}, \\
\left\|1-\mathcal{Q}^{j}\left(\overline{\mathcal{Q}}^{j}\right)^{-1}\right\|_{C^{0}\left(S^{1}, \mathbb{R}\right)},\left\|1-\overline{\mathcal{Q}}^{j}\left(\mathcal{Q}^{j}\right)^{-1}\right\|_{C^{0}\left(S^{1}, \mathbb{R}\right)} & \leq C H_{1}^{1 / 2}, \\
\left\|1-\mathcal{R}^{j}\left(\overline{\mathcal{R}}^{j}\right)^{-1}\right\|_{C^{0}\left(S^{1}, \mathbb{R}\right)},\left\|1-\overline{\mathcal{R}}^{j}\left(\mathcal{R}^{j}\right)^{-1}\right\|_{C^{0}\left(S^{1}, \mathbb{R}\right)} & \leq C H_{1}^{1 / 2}, \\
\left\|\left(\mathcal{Q}^{j}\right)^{1 / 2}\left(Q^{j}-\left\langle Q^{j}\right\rangle\right)\right\|_{C^{0}\left(S^{1}, \mathbb{R}\right)} & \leq C H_{1}^{1 / 2}, \\
\left\|\left(\mathcal{R}^{k}\right)^{1 / 2} \hat{B}^{k}\right\|_{C^{0}\left(S^{1}, \mathbb{R}\right)} & \leq C H_{1}^{1 / 2}, \\
\left(\overline{\mathcal{Q}}^{j}\right)^{1 / 2}\left|\left\langle Q_{t}^{j}\right\rangle\right| & \leq C H_{1, K}^{1 / 2}, \\
\int_{S^{1}} \mathcal{R}^{k}\left|\bar{A}^{k}\right|^{2} d \theta & \leq C H_{1, K}, \\
\int_{S^{1}} \mathcal{R}^{k}\left|\bar{B}^{k}\right|^{2} d \theta & \leq C H_{1} .
\end{aligned}
$$

Remark. The constants depend upon $H$, but they decrease as $H$ decreases. Note also that $H$ decreases with time.

Proof. The inequality (17) is obvious, and (18), (19) are immediate consequences of this. Note also that as a consequence of the first inequality, there are positive constants $c_{1}, c_{2}$ such that

$$
c_{1} \overline{\mathcal{Q}}^{j} \leq \mathcal{Q}^{j} \leq c_{2} \overline{\mathcal{Q}}^{j}, \quad c_{1} \overline{\mathcal{R}}^{j} \leq \mathcal{R}^{j} \leq c_{2} \overline{\mathcal{R}}^{j}
$$

for all $t \geq t_{0}$. Let us consider (20). We have

$$
\begin{aligned}
\left\|\left(\mathcal{Q}^{j}\right)^{1 / 2}\left(Q^{j}-\left\langle Q^{j}\right\rangle\right)\right\|_{C^{0}\left(S^{1}, \mathbb{R}\right)} & \leq C\left\|\left(\overline{\mathcal{Q}}^{j}\right)^{1 / 2}\left(Q^{j}-\left\langle Q^{j}\right\rangle\right)\right\|_{C^{0}\left(S^{1}, \mathbb{R}\right)} \\
& \leq C \int_{S^{1}}\left|\left(\overline{\mathcal{Q}}^{j}\right)^{1 / 2} Q_{\theta}^{j}\right| d \theta \\
& \leq C \int_{S^{1}}\left|\left(\mathcal{Q}^{j}\right)^{1 / 2} Q_{\theta}^{j}\right| d \theta \leq C\left[\int_{S^{1}} \mathcal{Q}^{j}\left(Q_{\theta}^{j}\right)^{2} d \theta\right]^{1 / 2} .
\end{aligned}
$$

Let us estimate

$$
\begin{aligned}
\int_{S^{1}} \overline{\mathcal{R}}^{k}\left(\bar{A}^{k}\right)^{2} d \theta \leq & \int_{S^{1}} \overline{\mathcal{R}}^{k} \bar{A}^{k} A^{k} d \theta \\
& +\sum_{j_{1}, j_{2}=1}^{m}\left|\alpha_{j_{1} j_{2}}^{k}\right| \int_{S^{1}} \overline{\mathcal{R}}^{k}\left|\left(Q^{j_{1}}-\left\langle Q^{j_{1}}\right\rangle\right) Q_{t}^{j_{2}} \bar{A}^{k}\right| d \theta \\
\leq & \int_{S^{1}} \overline{\mathcal{R}}^{k}\left(A^{k}\right)^{2} d \theta \\
& +\sum_{j_{1}, j_{2}=1}^{m}\left|\alpha_{j_{1} j_{2}}^{k}\right| \int_{S^{1}} \overline{\mathcal{R}}^{k}\left|\left(Q^{j_{1}}-\left\langle Q^{j_{1}}\right\rangle\right) Q_{t}^{j_{2}}\right|\left\{2\left|A^{k}\right|\right. \\
& \left.+\sum_{j_{1}^{\prime}, j_{2}^{\prime}=1}^{m}\left|\alpha_{j_{1}^{\prime} j_{2}^{\prime}}^{k}\left(Q^{j_{1}^{\prime}}-\left\langle Q^{j_{1}^{\prime}}\right\rangle\right) Q_{t}^{j_{2}^{\prime}}\right|\right\} d \theta \\
\leq & \int_{S^{1}} \mathcal{R}^{k}\left(A^{k}\right)^{2} d \theta+C H_{1}^{1 / 2} H_{1, K}+C H_{1} H_{1, K},
\end{aligned}
$$

where we have used (9), (19), (20), (25) and Hölder's inequality. Consequently, we obtain (23) after an applying (25). The proof of (24) is similar. Consider (21). We 
have

$$
\begin{aligned}
\left\|\left(\mathcal{R}^{k}\right)^{1 / 2} \hat{B}^{k}\right\|_{C^{0}\left(S^{1}, \mathbb{R}\right)} & \leq C\left\|\left(\overline{\mathcal{R}}^{k}\right)^{1 / 2} \hat{B}^{k}\right\|_{C^{0}\left(S^{1}, \mathbb{R}\right)} \\
& \leq C \int_{S^{1}}\left(\overline{\mathcal{R}}^{k}\right)^{1 / 2}\left|\bar{B}^{k}\right| d \theta \leq C H_{1}^{1 / 2}
\end{aligned}
$$

where we have used (24), (25) and Hölder's inequality. Estimate

$$
2 \pi\left|\left(\overline{\mathcal{Q}}^{j}\right)^{1 / 2}\left\langle Q_{t}^{j}\right\rangle\right| \leq \int_{S^{1}}\left(\overline{\mathcal{Q}}^{j}\right)^{1 / 2}\left|Q_{t}^{j}\right| d \theta \leq C \int_{S^{1}}\left(\mathcal{Q}^{j}\right)^{1 / 2}\left|Q_{t}^{j}\right| d \theta \leq C H_{K}^{1 / 2}
$$

where we used (25) and Hölder's inequality.

Define

$$
\Gamma^{1}=\frac{1}{2 t^{2}} \sum_{i=1}^{l} \int_{S^{1}}\left(P^{i}-\left\langle P^{i}\right\rangle\right) t P_{t}^{i} d \theta
$$

Lemma 2. Consider a solution to (12)-(15) and let $\Gamma^{1}$ be defined by (27). Then

$$
\left|\Gamma^{1}\right| \leq \frac{C}{t} H
$$

and

$$
\frac{d \Gamma^{1}}{d t} \leq-\frac{2}{t} \Gamma^{1}+\frac{1}{2 t} \int_{S^{1}}\left(\left|P_{t}\right|^{2}-\left|P_{\theta}\right|^{2}\right) d \theta+\frac{C}{t} H^{3 / 2} .
$$

Proof. The inequality (28) follows from (17) and the definition of $H$ and $\Gamma^{1}$. Let us compute

$$
\begin{aligned}
\frac{d \Gamma^{1}}{d t} & =-\frac{2}{t} \Gamma^{1}+\frac{1}{2 t} \int_{S^{1}}\left|P_{t}\right|^{2} d \theta-\frac{\pi}{t}\left|\left\langle P_{t}\right\rangle\right|^{2}+\frac{1}{2 t^{2}} \sum_{i=1}^{l} \int_{S^{1}}\left(P^{i}-\left\langle P^{i}\right\rangle\right) \partial_{t}\left(t P_{t}^{i}\right) d \theta \\
& \leq-\frac{2}{t} \Gamma^{1}+\frac{1}{2 t} \int_{S^{1}}\left|P_{t}\right|^{2} d \theta+\frac{1}{2 t^{2}} \sum_{i=1}^{l} \int_{S^{1}}\left(P^{i}-\left\langle P^{i}\right\rangle\right) t P_{\theta \theta}^{i} d \theta+\frac{C}{t} H^{3 / 2} \\
& \leq-\frac{2}{t} \Gamma^{1}+\frac{1}{2 t} \int_{S^{1}}\left(\left|P_{t}\right|^{2}-\left|P_{\theta}\right|^{2}\right) d \theta+\frac{C}{t} H^{3 / 2}
\end{aligned}
$$

where we have used (10), (13) and (17).

Let

$$
\Gamma^{2}=\frac{1}{2 t^{2}} \sum_{k=1}^{n} \int_{S^{1}} \overline{\mathcal{R}}^{k} \hat{B}^{k} t \bar{A}^{k} d \theta
$$

Lemma 3. Consider a solution to (12)-(15) and let $\Gamma^{2}$ be defined by (30). Then

$$
\left|\Gamma^{2}\right| \leq \frac{C}{t} H
$$

and

$$
\frac{d \Gamma^{2}}{d t} \leq-\frac{2}{t} \Gamma^{2}+\frac{1}{2 t} \sum_{k=1}^{n} \int_{S^{1}} \mathcal{R}^{k}\left[\left(A^{k}\right)^{2}-\left(B^{k}\right)^{2}\right] d \theta+\frac{C}{t} H^{3 / 2}
$$


Proof. Let us start by proving (31). Note first that

$$
\left\|\left(\overline{\mathcal{R}}^{k}\right)^{1 / 2} \hat{B}^{k}\right\|_{C^{0}\left(S^{1}, \mathbb{R}\right)} \leq C\left\|\left(\mathcal{R}^{k}\right)^{1 / 2} \hat{B}^{k}\right\|_{C^{0}\left(S^{1}, \mathbb{R}\right)} \leq C H^{1 / 2},
$$

where we have used (21) and (25). Consider

$$
\int_{S^{1}}\left(\overline{\mathcal{R}}^{k}\right)^{1 / 2}\left|\bar{A}^{k}\right| d \theta \leq C \int_{S^{1}}\left(\mathcal{R}^{k}\right)^{1 / 2}\left|\bar{A}^{k}\right| d \theta \leq C H^{1 / 2}
$$

by (23) and Hölder's inequality. Combining the above two inequalities, we obtain (31). Compute

$$
\begin{aligned}
\frac{d \Gamma^{2}}{d t}= & -\frac{2}{t} \Gamma^{2}+\frac{1}{2 t} \sum_{k=1}^{n} \int_{S^{1}} \sum_{i=1}^{l} \beta_{i}^{k}\left\langle P_{t}^{i}\right\rangle \overline{\mathcal{R}}^{k} \hat{B}^{k} \bar{A}^{k} d \theta+\frac{1}{2 t} \sum_{k=1}^{n} \int_{S^{1}} \overline{\mathcal{R}}^{k} \hat{B}_{t}^{k} \bar{A}^{k} d \theta \\
& +\frac{1}{2 t^{2}} \sum_{k=1}^{n} \int_{S^{1}} \overline{\mathcal{R}}^{k} \hat{B}^{k} \partial_{t}\left(t \bar{A}^{k}\right) d \theta .
\end{aligned}
$$

Note that $\left|\left\langle P_{t}^{i}\right\rangle\right| \leq C H^{1 / 2}$, so that

$$
\left|\frac{1}{2 t} \sum_{k=1}^{n} \int_{S^{1}} \sum_{i=1}^{l} \beta_{i}^{k}\left\langle P_{t}^{i}\right\rangle \overline{\mathcal{R}}^{k} \hat{B}^{k} \bar{A}^{k} d \theta\right| \leq \frac{C}{t} H^{3 / 2}
$$

where the argument to prove the last inequality is the same as the proof of (31). Compute

$$
\hat{B}_{t}^{k}=\bar{A}^{k}-\left\langle\bar{A}^{k}\right\rangle+\sum_{j_{1}, j_{2}=1}^{m} \alpha_{j_{1} j_{2}}^{k}\left\langle Q_{t}^{j_{1}}\right\rangle\left(Q^{j_{2}}-\left\langle Q^{j_{2}}\right\rangle\right)
$$

Note that

$$
\sum_{j_{1}, j_{2}=1}^{m} \alpha_{j_{1} j_{2}}^{k} \int_{S^{1}} \overline{\mathcal{R}}^{k}\left\langle Q_{t}^{j_{1}}\right\rangle\left(Q^{j_{2}}-\left\langle Q^{j_{2}}\right\rangle\right) \bar{A}^{k} d \theta \leq C H^{3 / 2}
$$

by (9), (20), (22), (23), (25) and Hölder's inequality. Since we also have

$$
\int_{S^{1}} \overline{\mathcal{R}}^{k}\left(\bar{A}^{k}-\left\langle\bar{A}^{k}\right\rangle\right) \bar{A}^{k} d \theta \leq \int_{S^{1}} \overline{\mathcal{R}}^{k}\left(\bar{A}^{k}\right)^{2} d \theta-2 \pi \overline{\mathcal{R}}^{k}\left\langle\bar{A}^{k}\right\rangle^{2},
$$

the relevant object to study is

$$
\int_{S^{1}} \overline{\mathcal{R}}^{k}\left(\bar{A}^{k}\right)^{2} d \theta \leq \int_{S^{1}} \mathcal{R}^{k}\left(A^{k}\right)^{2} d \theta+C H^{3 / 2},
$$

where we have used (26). Adding up the above observations, we get

$$
\frac{d \Gamma^{2}}{d t} \leq-\frac{2}{t} \Gamma^{2}+\frac{1}{2 t} \sum_{k=1}^{n} \int_{S^{1}} \mathcal{R}^{k}\left(A^{k}\right)^{2} d \theta+\frac{1}{2 t^{2}} \sum_{k=1}^{n} \int_{S^{1}} \overline{\mathcal{R}}^{k} \hat{B}^{k} \partial_{t}\left(t \bar{A}^{k}\right) d \theta+\frac{C}{t} H^{3 / 2}
$$

Note that

$$
\partial_{t}\left(t A^{k}\right)-\partial_{t}\left(t \bar{A}^{k}\right)=\sum_{j_{1}, j_{2}=1}^{m} \alpha_{j_{1} j_{2}}^{k}\left[\left(Q_{t}^{j_{1}}-\left\langle Q_{t}^{j_{1}}\right\rangle\right) t Q_{t}^{j_{2}}+\left(Q^{j_{1}}-\left\langle Q^{j_{1}}\right\rangle\right) \partial_{t}\left(t Q_{t}^{j_{2}}\right)\right]
$$

Estimate

$$
\left|\alpha_{j_{1} j_{2}}^{k} \int_{S^{1}} \overline{\mathcal{R}}^{k} \hat{B}^{k}\left(Q_{t}^{j_{1}}-\left\langle Q_{t}^{j_{1}}\right\rangle\right) t Q_{t}^{j_{2}} d \theta\right| \leq C t H^{3 / 2}
$$


where we have used (9), (21), (22), (25), and Hölder's inequality. Estimate

$$
\begin{aligned}
\mid \alpha_{j_{1} j_{2}}^{k} & \int_{S^{1}} \overline{\mathcal{R}}^{k} \hat{B}^{k}\left(Q^{j_{1}}-\left\langle Q^{j_{1}}\right\rangle\right) \partial_{t}\left(t Q_{t}^{j_{2}}\right) d \theta \mid \\
= & \mid \alpha_{j_{1} j_{2}}^{k} \int_{S^{1}} \overline{\mathcal{R}}^{k} \hat{B}^{k}\left(Q^{j_{1}}-\left\langle Q^{j_{1}}\right\rangle\right)\left(\mathcal{Q}^{j_{2}}\right)^{-1}\left[\partial_{t}\left(t \mathcal{Q}^{j_{2}} Q_{t}^{j_{2}}\right)\right. \\
& \left.\quad-t \sum_{i=1}^{l} \alpha_{i}^{j_{2}} P_{t}^{i} \mathcal{Q}^{j_{2}} Q_{t}^{j_{2}}\right] d \theta \mid \\
\leq & \left|\alpha_{j_{1} j_{2}}^{k} \int_{S^{1}} \overline{\mathcal{R}}^{k} \hat{B}^{k}\left(Q^{j_{1}}-\left\langle Q^{j_{1}}\right\rangle\right)\left(\mathcal{Q}^{j_{2}}\right)^{-1} \partial_{t}\left(t \mathcal{Q}^{j_{2}} Q_{t}^{j_{2}}\right) d \theta\right|+C t H^{2} .
\end{aligned}
$$

Consider (14). Let us estimate

$$
\left|\alpha_{j_{1} j_{2}}^{k} \int_{S^{1}} \overline{\mathcal{R}}^{k} \hat{B}^{k}\left(Q^{j_{1}}-\left\langle Q^{j_{1}}\right\rangle\right)\left(\mathcal{Q}^{j_{2}}\right)^{-1} \partial_{\theta}\left(t \mathcal{Q}^{j_{2}} Q_{\theta}^{j_{2}}\right) d \theta\right| \leq C t H^{3 / 2}
$$

by arguments similar to ones given above, after one has carried out a partial integration. Some of the remaining terms in (14) give rise to terms of the form

$$
\begin{aligned}
& \left|\int_{S^{1}} \overline{\mathcal{R}}^{k} \hat{B}^{k}\left(Q^{j_{1}}-\left\langle Q^{j_{1}}\right\rangle\right)\left(\mathcal{Q}^{j_{2}}\right)^{-1} t \mathcal{R}^{k^{\prime}}\left(Q_{t}^{o} A^{k^{\prime}}-Q_{\theta}^{o} B^{k^{\prime}}\right) d \theta\right| \\
& \quad \leq C t \int_{S^{1}}\left|\left(\mathcal{R}^{k}\right)^{1 / 2} \hat{B}^{k}\left(\mathcal{Q}^{j_{1}}\right)^{1 / 2}\left(Q^{j_{1}}-\left\langle Q^{j_{1}}\right\rangle\right)\left(\mathcal{Q}^{o}\right)^{1 / 2}\left(\mathcal{R}^{k^{\prime}}\right)^{1 / 2}\left(Q_{t}^{o} A^{k^{\prime}}-Q_{\theta}^{o} B^{k^{\prime}}\right)\right| d \theta \\
& \quad \leq C t H^{2},
\end{aligned}
$$

where we have used the facts that $\mathcal{R}^{k^{\prime}}=\mathcal{Q}^{j_{2}} \mathcal{Q}^{o}$ and $\mathcal{R}^{k}=\mathcal{Q}^{j_{2}} \mathcal{Q}^{j_{1}}$ whenever terms of the above form appear. Finally, we have to estimate

$$
\left|\alpha_{j_{1} j_{2}}^{k} \int_{S^{1}} \overline{\mathcal{R}}^{k} \hat{B}^{k}\left(Q^{j_{1}}-\left\langle Q^{j_{1}}\right\rangle\right)\left(\mathcal{Q}^{j_{2}}\right)^{-1} \frac{\partial \bar{h}_{\gamma \delta}}{\partial Q^{j_{2}}}\left(g_{t}^{\gamma} g_{t}^{\delta}-g_{\theta}^{\gamma} g_{\theta}^{\delta}\right)\right| \leq C t H^{2},
$$

where we have used (9), (11), (20), (21) and (25). We conclude that

$$
\left|\alpha_{j_{1} j_{2}}^{k} \int_{S^{1}} \overline{\mathcal{R}}^{k} \hat{B}^{k}\left(Q^{j_{1}}-\left\langle Q^{j_{1}}\right\rangle\right) \partial_{t}\left(t Q_{t}^{j_{2}}\right) d \theta\right| \leq C t H^{3 / 2}
$$

Thus

$$
\frac{1}{2 t^{2}} \sum_{k=1}^{n} \int_{S^{1}} \overline{\mathcal{R}}^{k} \hat{B}^{k} \partial_{t}\left(t \bar{A}^{k}\right) d \theta \leq \frac{1}{2 t^{2}} \sum_{k=1}^{n} \int_{S^{1}} \overline{\mathcal{R}}^{k} \hat{B}^{k} \partial_{t}\left(t A^{k}\right) d \theta+\frac{C}{t} H^{3 / 2} .
$$

Let us estimate

$$
\begin{aligned}
\int_{S^{1}} \overline{\mathcal{R}}^{k} \hat{B}^{k} \partial_{t}\left(t A^{k}\right) d \theta & =\int_{S^{1}} \overline{\mathcal{R}}^{k} \hat{B}^{k}\left(\mathcal{R}^{k}\right)^{-1}\left[\partial_{t}\left(t \mathcal{R}^{k} A^{k}\right)-t \sum_{i=1}^{l} \beta_{i}^{k} P_{t}^{i} \mathcal{R}^{k} A^{k}\right] d \theta \\
& \leq \int_{S^{1}} \overline{\mathcal{R}}^{k} \hat{B}^{k}\left(\mathcal{R}^{k}\right)^{-1} \partial_{\theta}\left(t \mathcal{R}^{k} B^{k}\right) d \theta+C t H^{3 / 2} \\
& \leq \sum_{i=1}^{l} \beta_{i}^{k} t \int_{S^{1}} \overline{\mathcal{R}}^{k} \hat{B}^{k} P_{\theta}^{i} B^{k} d \theta-\int_{S^{1}} t \overline{\mathcal{R}}^{k} \bar{B}^{k} B^{k} d \theta+C t H^{3 / 2} \\
& \leq-\int_{S^{1}} t \mathcal{R}^{k}\left(B^{k}\right)^{2} d \theta+C t H^{3 / 2},
\end{aligned}
$$


where the arguments to prove the last inequality are standard by now. We conclude that

$$
\frac{1}{2 t^{2}} \sum_{k=1}^{n} \int_{S^{1}} \overline{\mathcal{R}}^{k} \hat{B}^{k} \partial_{t}\left(t \bar{A}^{k}\right) d \theta \leq-\frac{1}{2 t} \sum_{k=1}^{n} \int_{S^{1}} \mathcal{R}^{k}\left(B^{k}\right)^{2} d \theta+\frac{C}{t} H^{3 / 2} .
$$

The lemma follows.

Define

$$
\Gamma^{3}=\frac{1}{2 t^{2}} \sum_{j=1}^{m} \int_{S^{1}} \overline{\mathcal{Q}}^{j}\left(Q^{j}-\left\langle Q^{j}\right\rangle\right) t Q_{t}^{j} d \theta
$$

Lemma 4. Consider a solution to (12)-(15) and let $\Gamma^{3}$ be defined by (33). Then

$$
\left|\Gamma^{3}\right| \leq \frac{C}{t} H
$$

and

$$
\frac{d \Gamma^{3}}{d t} \leq-\frac{2}{t} \Gamma^{3}+\frac{1}{2 t} \sum_{j=1}^{m} \int_{S^{1}} \mathcal{Q}^{j}\left[\left(Q_{t}^{j}\right)^{2}-\left(Q_{\theta}^{j}\right)^{2}\right] d \theta+\frac{C}{t} H^{3 / 2}
$$

Proof. The inequality (34) follows from (20), (25) and Hölder's inequality. Similarly to earlier arguments, we have

$\frac{d \Gamma^{3}}{d t} \leq-\frac{2}{t} \Gamma^{3}+\frac{1}{2 t} \sum_{j=1}^{m} \int_{S^{1}} \mathcal{Q}^{j}\left(Q_{t}^{j}\right)^{2} d \theta+\frac{1}{2 t^{2}} \sum_{j=1}^{m} \int_{S^{1}} \overline{\mathcal{Q}}^{j}\left(Q^{j}-\left\langle Q^{j}\right\rangle\right) \partial_{t}\left(t Q_{t}^{j}\right) d \theta+\frac{C}{t} H^{3 / 2}$.

Consider

$$
\begin{aligned}
\int_{S^{1}} \overline{\mathcal{Q}}^{j}\left(Q^{j}-\left\langle Q^{j}\right\rangle\right) \partial_{t}\left(t Q_{t}^{j}\right) d \theta= & \int_{S^{1}} \overline{\mathcal{Q}}^{j}\left(Q^{j}-\left\langle Q^{j}\right\rangle\right)\left(\mathcal{Q}^{j}\right)^{-1}\left[\partial_{t}\left(t \mathcal{Q}^{j} Q_{t}^{j}\right)\right. \\
& \left.-t \sum_{i=1}^{l} \alpha_{i}^{j} P_{t}^{i} \mathcal{Q}^{j} Q_{t}^{j}\right] d \theta \\
\leq & \int_{S^{1}} \overline{\mathcal{Q}}^{j}\left(Q^{j}-\left\langle Q^{j}\right\rangle\right)\left(\mathcal{Q}^{j}\right)^{-1} \partial_{t}\left(t \mathcal{Q}^{j} Q_{t}^{j}\right) d \theta \\
& +C t H^{3 / 2}
\end{aligned}
$$

Let us use (14). We need the estimate

$$
\left|\int_{S^{1}} \overline{\mathcal{Q}}^{j}\left(Q^{j}-\left\langle Q^{j}\right\rangle\right)\left(\mathcal{Q}^{j}\right)^{-1} t \mathcal{R}^{k}\left(Q_{t}^{o} A^{k}-Q_{\theta}^{o} B^{k}\right) d \theta\right| \leq C t H^{3 / 2},
$$

where we have used the fact that $\mathcal{R}^{k}=\mathcal{Q}^{j} \mathcal{Q}^{o}$ whenever terms of the above form appear. Furthermore, we have

$$
\left|\int_{S^{1}} \overline{\mathcal{Q}}^{j}\left(Q^{j}-\left\langle Q^{j}\right\rangle\right)\left(\mathcal{Q}^{j}\right)^{-1} \frac{\partial \bar{h}_{\gamma \delta}}{\partial Q^{j}}\left(g_{t}^{\gamma} g_{t}^{\delta}-g_{\theta}^{\gamma} g_{\theta}^{\delta}\right) d \theta\right| \leq C t H^{3 / 2},
$$


where we have used (11), (20) and (25). What remains to be considered is then

$$
\begin{aligned}
& \int_{S^{1}} \overline{\mathcal{Q}}^{j}\left(Q^{j}-\left\langle Q^{j}\right\rangle\right)\left(\mathcal{Q}^{j}\right)^{-1} \partial_{\theta}\left(t \mathcal{Q}^{j} Q_{\theta}^{j}\right) d \theta \\
& \quad=-\int_{S^{1}} t \overline{\mathcal{Q}}^{j}\left(Q_{\theta}^{j}\right)^{2} d \theta+\sum_{i=1}^{l} \alpha_{i}^{j} t \int_{S^{1}} \overline{\mathcal{Q}}^{j}\left(Q^{j}-\left\langle Q^{j}\right\rangle\right) P_{\theta}^{i} Q_{\theta}^{j} d \theta \\
& \quad \leq-\int_{S^{1}} t \mathcal{Q}^{j}\left(Q_{\theta}^{j}\right)^{2} d \theta+C t H^{3 / 2}
\end{aligned}
$$

Adding up, we get

$$
\frac{1}{2 t^{2}} \sum_{j=1}^{m} \int_{S^{1}} \overline{\mathcal{Q}}^{j}\left(Q^{j}-\left\langle Q^{j}\right\rangle\right) \partial_{t}\left(t Q_{t}^{j}\right) d \theta \leq-\frac{1}{2 t} \sum_{j=1}^{m} \int_{S^{1}} \mathcal{Q}^{j}\left(Q_{\theta}^{j}\right)^{2} d \theta+\frac{C}{t} H^{3 / 2} .
$$

The lemma follows.

Proof of Proposition 1. By (4), we know that $H$ is monotonically decaying, so we shall assume $t_{0} \geq 1$. Furthermore, all constants depend on $H$, but they decrease as $H$ decreases. Consequently, if we demand that $\epsilon \leq 1$, all the constants that depend on $H$ become numerical constants. Define

$$
\Gamma=\Gamma^{1}+\Gamma^{2}+\Gamma^{3}
$$

By (28), (31) and (34), we have

$$
|\Gamma| \leq \frac{C}{t} H
$$

where $C$ depends on $H$. By (4), (29), (32), (35) and (36), we have

$$
\frac{d(H+\Gamma)}{d t} \leq-\frac{1}{t}(H+\Gamma)+\frac{C}{t^{2}} H+\frac{C}{t} H^{3 / 2}
$$

for $t \geq t_{0}$. Due to (36) there is a $T \geq t_{0}$ such that

$$
\frac{1}{2} H \leq H+\Gamma \leq 2 H
$$

for $t \geq T$. Thus, if we let $\mathcal{E}=H+\Gamma$, then

$$
\frac{d \mathcal{E}}{d t} \leq-\frac{1}{t} \mathcal{E}+\frac{C}{t^{2}} \mathcal{E}+\frac{C}{t} \mathcal{E}^{3 / 2}
$$

for $t \geq T$. For $\epsilon$ small enough, $C \mathcal{E}^{1 / 2}(t) \leq 1 / 2$ for all $t \geq T$. Then

$$
\frac{d \mathcal{E}}{d t} \leq-\frac{1}{2 t} \mathcal{E}+\frac{C}{t^{2}} \mathcal{E}
$$

so that $\mathcal{E} \leq C t^{-1 / 2}$. Inserting this information into (37), we get the conclusion that

$$
\frac{d \mathcal{E}}{d t} \leq\left(-\frac{1}{t}+\frac{C}{t^{2}}+\frac{C}{t^{5 / 4}}\right) \mathcal{E}
$$

and the proposition follows. 


\section{LARGE DATA}

In this section it will be necessary to make the additional assumption that the metric is ordered. We shall use the notation

$$
J_{1}=\left\{j_{1}: \exists k, j_{2}: \alpha_{j_{1} j_{2}}^{k} \neq 0\right\} .
$$

Note that if $j_{1} \in J_{1}$, then $\alpha_{j_{2} j_{1}}^{k}=0$ for all $j_{2}$ and $k$ (assuming that the metric $\bar{g}$ of the form (8) is ordered). In the proof of Theorem 1 , it will be convenient to know the following facts.

Lemma 5. Consider a solution to (12)-(15). Then

$$
\begin{aligned}
\frac{1}{t} \int_{S^{1}} P_{t} \cdot\left\langle P_{t}\right\rangle d \theta & \in L^{1}\left(\left[t_{0}, \infty\right)\right) \\
\frac{1}{t^{2}} \int_{S^{1}} \mathcal{Q}^{j} Q_{t}^{j}\left(Q^{j}-\left\langle Q^{j}\right\rangle\right) d \theta & \in L^{1}\left(\left[t_{0}, \infty\right)\right) \\
\frac{1}{t} \int_{S^{1}} \mathcal{R}^{k} A^{k}\left\langle\bar{A}^{k}\right\rangle d \theta & \in L^{1}\left(\left[t_{0}, \infty\right)\right) \\
\frac{1}{t} \alpha_{j_{1} j_{2}}^{k} \int_{S^{1}} \mathcal{R}^{k} A^{k}\left\langle Q_{t}^{j_{1}}\right\rangle\left(Q^{j_{2}}-\left\langle Q^{j_{2}}\right\rangle\right) d \theta & \in L^{1}\left(\left[t_{0}, \infty\right)\right) \\
\frac{1}{t} \alpha_{j_{1} j_{2}}^{k} \int_{S^{1}} \mathcal{R}^{k} A^{k}\left(Q^{j_{1}}-\left\langle Q^{j_{1}}\right\rangle\right) Q_{t}^{j_{2}} d \theta & \in L^{1}\left(\left[t_{0}, \infty\right)\right)
\end{aligned}
$$

for all $t_{0}>0$

Proof. Note that (4) implies that

$$
\frac{1}{t} H_{1, K}+\frac{1}{t} H_{2} \in L^{1}\left(\left[t_{0}, \infty\right)\right)
$$

for every $t_{0}>0$. Estimate

$$
\frac{1}{t}\left|\int_{S^{1}} P_{t} \cdot\left\langle P_{t}\right\rangle d \theta\right|=\frac{2 \pi}{t}\left|\left\langle P_{t}\right\rangle\right|^{2} \leq \frac{1}{t} \int_{S^{1}}\left|P_{t}\right|^{2} d \theta \leq \frac{2}{t} H_{1, K},
$$

proving (38) due to (43). Note that

$$
\left|\int_{S^{1}} \mathcal{Q}^{j} Q_{t}^{j}\left(Q^{j}-\left\langle Q^{j}\right\rangle\right) d \theta\right| \leq C H_{1}
$$

by (20) and Hölder's inequality. Since $H$ is bounded to the future, we have (39). Estimate

$$
\left|\int_{S^{1}} \mathcal{R}^{k} A^{k}\left\langle\bar{A}^{k}\right\rangle d \theta\right| \leq C\left|\int_{S^{1}}\left(\mathcal{R}^{k}\right)^{1 / 2}\right| A^{k}|d \theta|\left|\int_{S^{1}}\left(\mathcal{R}^{k}\right)^{1 / 2}\right| \bar{A}^{k}|d \theta| \leq C H_{1, K},
$$

where we have used (25), (23) and Hölder's inequality. We conclude that (40) holds since (43) is true. The statements (41) and (42) can be proven by similar methods after taking (9) into account.

The statements of the above lemma, and similar results which have not been stated, will be used without further comment in the following.

Proof of Theorem 1. Due to Proposition 1, all we need to prove is that $H$ converges to zero. One way of doing so is to prove that $t^{-1} H \in L^{1}\left(\left[t_{0}, \infty\right)\right)$ for some $t_{0}>0$. In fact, since $H$ is monotonically decaying, it has to converge to some $c_{0} \geq 0$, and if $c_{0}>0$, we get a contradiction to the integrability of $t^{-1} H$. Note that we have 
(43), so that all we need to prove is that $t^{-1} H_{1, P} \in L^{1}\left(\left[t_{0}, \infty\right)\right)$ for some $t_{0}>0$. Let us compute

$$
\begin{aligned}
\int_{t_{0}}^{t} \frac{1}{s} \int_{S^{1}} \mathcal{Q}^{j}\left[\left(Q_{t}^{j}\right)^{2}-\left(Q_{\theta}^{j}\right)^{2}\right] d \theta d s \\
=\int_{t_{0}}^{t} \frac{1}{s} \int_{S^{1}} \mathcal{Q}^{j}\left[Q_{t}^{j} \partial_{t}\left(Q^{j}-\left\langle Q^{j}\right\rangle\right)-\left(Q_{\theta}^{j}\right)^{2}\right] d \theta d s+\ldots \\
=-\int_{t_{0}}^{t} \frac{1}{s^{2}} \int_{S^{1}}\left[\partial_{t}\left(s \mathcal{Q}^{j} Q_{t}^{j}\right)-\partial_{\theta}\left(s \mathcal{Q}^{j} Q_{\theta}^{j}\right)\right]\left(Q^{j}-\left\langle Q^{j}\right\rangle\right) d \theta d s+\ldots \\
=\int_{t_{0}}^{t} \frac{1}{s} \int_{S^{1}} \sum_{k=1}^{n} \mathcal{R}^{k} \sum_{o=1}^{m}\left(\alpha_{o j}^{k}-\alpha_{j o}^{k}\right)\left(Q_{t}^{o} A^{k}-Q_{\theta}^{o} B^{k}\right)\left(Q^{j}-\left\langle Q^{j}\right\rangle\right) d \theta d s \\
\quad-\int_{t_{0}}^{t} \frac{1}{s^{2}} \int_{S^{1}} \frac{1}{2} \frac{\partial \bar{h}_{\gamma \delta}}{\partial Q^{j}}\left(g_{t}^{\gamma} g_{t}^{\delta}-g_{\theta}^{\gamma} g_{\theta}^{\delta}\right)\left(Q^{j}-\left\langle Q^{j}\right\rangle\right) d \theta d s+\ldots \\
=-\int_{t_{0}}^{t} \frac{1}{s} \int_{S^{1}} \sum_{k=1}^{n} \mathcal{R}^{k} \sum_{o=1}^{m}\left(\alpha_{o j}^{k}-\alpha_{j o}^{k}\right) Q_{\theta}^{o} B^{k}\left(Q^{j}-\left\langle Q^{j}\right\rangle\right) d \theta d s+\ldots
\end{aligned}
$$

Here, and below, ... represents functions of $t$ that converge to some finite number as $t \rightarrow \infty$. Note in particular that the terms that arise from the endpoints $t_{0}$ and $t$ when carrying out a partial integration with respect to $s$ fall into this class. Consider

Note that

$$
\int_{t_{0}}^{t} \frac{1}{s} \int_{S^{1}} \mathcal{R}^{k}\left[\left(A^{k}\right)^{2}-\left(B^{k}\right)^{2}\right] d \theta d s .
$$

$$
\begin{aligned}
A^{k}= & \hat{B}_{t}^{k}+\left\langle\bar{A}^{k}\right\rangle-\sum_{j_{1}, j_{2}=1}^{m} \alpha_{j_{1} j_{2}}^{k}\left\langle Q_{t}^{j_{1}}\right\rangle\left(Q^{j_{2}}-\left\langle Q^{j_{2}}\right\rangle\right) \\
& +\sum_{j_{1}, j_{2}=1}^{m} \alpha_{j_{1} j_{2}}^{k}\left(Q^{j_{1}}-\left\langle Q^{j_{1}}\right\rangle\right) Q_{t}^{j_{2}} .
\end{aligned}
$$

This can be used to conclude that

$$
\begin{aligned}
\int_{t_{0}}^{t} \frac{1}{s} \int_{S^{1}} \mathcal{R}^{k}\left(A^{k}\right)^{2} d \theta d s & =\int_{t_{0}}^{t} \frac{1}{s} \int_{S^{1}} \mathcal{R}^{k} A^{k} \hat{B}_{t}^{k} d \theta d s+\ldots \\
& =-\int_{t_{0}}^{t} \frac{1}{s^{2}} \int_{S^{1}} \partial_{t}\left(s \mathcal{R}^{k} A^{k}\right) \hat{B}^{k} d \theta d s+\ldots
\end{aligned}
$$

Note that

$$
B^{k}=\hat{B}_{\theta}^{k}+\sum_{j_{1}, j_{2}=1}^{m} \alpha_{j_{1} j_{2}}^{k}\left(Q^{j_{1}}-\left\langle Q^{j_{1}}\right\rangle\right) Q_{\theta}^{j_{2}}
$$

so that

$$
\begin{aligned}
- & \int_{t_{0}}^{t} \frac{1}{s} \int_{S^{1}} \mathcal{R}^{k}\left(B^{k}\right)^{2} d \theta d s \\
= & \int_{t_{0}}^{t} \frac{1}{s^{2}} \int_{S^{1}} \partial_{\theta}\left(s \mathcal{R}^{k} B^{k}\right) \hat{B}^{k} d \theta d s \\
& -\sum_{j_{1}, j_{2}=1}^{m} \alpha_{j_{1} j_{2}}^{k} \int_{t_{0}}^{t} \frac{1}{s} \int_{S^{1}} \mathcal{R}^{k} B^{k}\left(Q^{j_{1}}-\left\langle Q^{j_{1}}\right\rangle\right) Q_{\theta}^{j_{2}} d \theta d s
\end{aligned}
$$


Using (12), we get

$$
\begin{aligned}
& \int_{t_{0}}^{t} \frac{1}{s} \int_{S^{1}} \mathcal{R}^{k}\left[\left(A^{k}\right)^{2}-\left(B^{k}\right)^{2}\right] d \theta d s \\
& \quad=-\sum_{j_{1}, j_{2}=1}^{m} \alpha_{j_{1} j_{2}}^{k} \int_{t_{0}}^{t} \frac{1}{s} \int_{S^{1}} \mathcal{R}^{k} B^{k}\left(Q^{j_{1}}-\left\langle Q^{j_{1}}\right\rangle\right) Q_{\theta}^{j_{2}} d \theta d s+\ldots
\end{aligned}
$$

Summing (44) over $j \in J_{1}$, we obtain

$$
\begin{aligned}
\sum_{j_{1} \in J_{1}} \int_{t_{0}}^{t} \frac{1}{s} \int_{S^{1}} \mathcal{Q}^{j_{1}}\left[\left(Q_{t}^{j_{1}}\right)^{2}-\left(Q_{\theta}^{j_{1}}\right)^{2}\right] d \theta d s \\
\quad=\sum_{j_{1} \in J_{1}} \sum_{k=1}^{n} \sum_{j_{2}=1}^{m} \int_{t_{0}}^{t} \frac{1}{s} \int_{S^{1}} \mathcal{R}^{k} \alpha_{j_{1} j_{2}}^{k} Q_{\theta}^{j_{2}} B^{k}\left(Q^{j_{1}}-\left\langle Q^{j_{1}}\right\rangle\right) d \theta d s+\ldots
\end{aligned}
$$

Summing (45) over $k$, we obtain

$$
\begin{aligned}
& \sum_{k=1}^{n} \int_{t_{0}}^{t} \frac{1}{s} \int_{S^{1}} \mathcal{R}^{k}\left[\left(A^{k}\right)^{2}-\left(B^{k}\right)^{2}\right] d \theta d s \\
& \quad=-\sum_{j_{1} \in J_{1}} \sum_{k=1}^{n} \sum_{j_{2}=1}^{m} \alpha_{j_{1} j_{2}}^{k} \int_{t_{0}}^{t} \frac{1}{s} \int_{S^{1}} \mathcal{R}^{k} B^{k}\left(Q^{j_{1}}-\left\langle Q^{j_{1}}\right\rangle\right) Q_{\theta}^{j_{2}} d \theta d s+\ldots
\end{aligned}
$$

In other words

$$
\int_{t_{0}}^{t} \frac{1}{s} \int_{S^{1}}\left\{\sum_{k=1}^{n} \mathcal{R}^{k}\left[\left(A^{k}\right)^{2}-\left(B^{k}\right)^{2}\right]+\sum_{j \in J_{1}} \mathcal{Q}^{j_{1}}\left[\left(Q_{t}^{j_{1}}\right)^{2}-\left(Q_{\theta}^{j_{1}}\right)^{2}\right]\right\} d \theta d s=\ldots
$$

We conclude that

$$
\frac{1}{t} \int_{S^{1}}\left\{\sum_{k=1}^{n} \mathcal{R}^{k}\left(B^{k}\right)^{2}+\sum_{j_{1} \in J_{1}} \mathcal{Q}^{j_{1}}\left(Q_{\theta}^{j_{1}}\right)^{2}\right\} d \theta \in L^{1}\left(\left[t_{0}, \infty\right)\right)
$$

for all $t_{0}>0$. Consider (44). Estimate

$$
\frac{\left|\alpha_{o j}^{k}\right|}{t} \int_{S^{1}} \mathcal{R}^{k}\left|Q_{\theta}^{o} B^{k}\left(Q^{j}-\left\langle Q^{j}\right\rangle\right)\right| d \theta \leq \frac{C}{t} \int_{S^{1}}\left\{\sum_{k=1}^{n} \mathcal{R}^{k}\left(B^{k}\right)^{2}+\sum_{j_{1} \in J_{1}} \mathcal{Q}^{j_{1}}\left(Q_{\theta}^{j_{1}}\right)^{2}\right\} d \theta
$$

by Hölder's inequality since $o \in J_{1}$ and (9) and (20) hold. Since

$$
\|\left(\mathcal{Q}^{j}\right)^{1 / 2}\left(Q^{j}-\left\langle Q^{j}\right\rangle \|_{C^{0}\left(S^{1}, \mathbb{R}\right)} \leq C\left[\int_{S^{1}} \mathcal{Q}^{j}\left(Q_{\theta}^{j}\right)^{2} d \theta\right]^{1 / 2},\right.
$$

cf. the proof of (20), we can proceed similarly to the above in order to prove that $\frac{\left|\alpha_{j o}^{k}\right|}{t} \int_{S^{1}} \mathcal{R}^{k}\left|Q_{\theta}^{o} B^{k}\left(Q^{j}-\left\langle Q^{j}\right\rangle\right)\right| d \theta \leq \frac{C}{t} \int_{S^{1}}\left\{\sum_{k=1}^{n} \mathcal{R}^{k}\left(B^{k}\right)^{2}+\sum_{j_{1} \in J_{1}} \mathcal{Q}^{j_{1}}\left(Q_{\theta}^{j_{1}}\right)^{2}\right\} d \theta$.

This information can then be used together with (44) and (46) in order to prove that

$$
\frac{1}{t} \int_{S^{1}}\left\{\sum_{k=1}^{n} \mathcal{R}^{k}\left(B^{k}\right)^{2}+\sum_{j=1}^{m} \mathcal{Q}^{j}\left(Q_{\theta}^{j}\right)^{2}\right\} d \theta \in L^{1}\left(\left[t_{0}, \infty\right)\right)
$$


Finally let us consider

$$
\begin{gathered}
\int_{t_{0}}^{t} \frac{1}{s} \int_{S^{1}}\left[\left|P_{t}\right|^{2}-\left|P_{\theta}\right|^{2}\right] d \theta d s=-\int_{t_{0}}^{t} \frac{1}{s^{2}} \int_{S^{1}}\left[\partial_{t}\left(s P_{t}\right)-\partial_{\theta}\left(s P_{\theta}\right)\right] \cdot(P-\langle P\rangle) d \theta d s \\
+\ldots=\ldots,
\end{gathered}
$$

where we have used (10), (13), (43) and (47) in order to obtain the last equality. Due to this fact, (43) and (47), we conclude that $t^{-1} H \in L^{1}\left(\left[t_{0}, \infty\right)\right)$, and the theorem follows.

\section{Conclusions}

In this paper, we have demonstrated that some of the arguemts of [6] can be generalized to other situations. One rather fundamental question has however not been answered. Namely, what is the property of the target space (in the case $\mathcal{L}_{1}=0$ and the problem is of wave map type) that implies the decay of the energy? What we would like to have is of course a geometric condition. It is interesting to note that the arguments are not very geometric. Consider for instance the proof of Theorem 1. Due to (4), we know that $t^{-1} H_{1, K}+t^{-1} H_{2} \in L^{1}\left(\left[t_{0}, \infty\right)\right)$ for all $t_{0}>0$. Geometrically, it would then seem natural to consider the limit of

$$
\int_{t_{0}}^{t} \frac{1}{s}\left(H_{1, K}-H_{1, P}\right) d s
$$

as $t \rightarrow \infty$. It is however not clear to us how to do something sensible with this expression. Instead, we first prove (46) and then proceed to consider the other parts of the potential energy. In other words, we divide the potential energy into different parts, without there being any clear geometric interpretation of this division, and then successively arrive at the desired conclusion. Note also that it is here that the condition that the metric be ordered comes in. Finally, let us observe that the presence or absence of $\mathcal{L}_{2}$ in the Lagrangian density (assuming that $\bar{h}$ is a $\bar{g}$-metric) does not make any significant difference as far as the complexity of the argument is concerned.

\section{REFERENCES}

[1] Chruściel P T 1990 On spacetimes with $U(1) \times U(1)$ symmetric compact Cauchy surfaces $A n n$. Phys. NY 202 100-50

[2] Gowdy R H 1974 Vacuum spacetimes with two-parameter spacelike isometry groups and compact invariant hypersurfaces: Topologies and boundary conditions Ann. Phys. NY 83 203-41

[3] Moncrief V 1981 Global properties of Gowdy spacetimes with $T^{3} \times \mathbb{R}$ topology Ann. Phys. NY 132 87-107

[4] Narita M 2003 Global existence problem in $T^{3}$-Gowdy symmetric IIB superstring cosmology Class. Quantum Grav. 20 4983-94

[5] Narita M 2002 On the existence of global solutions for $T^{3}$-Gowdy spacetimes with stringy matter Class. Quantum Grav. $196279-88$

[6] Ringström H 2004 On a wave map equation arising in General Relativity Commun Pure Appl Math 57 657-703

Max-Planck-Institut für Gravitationsphysik, Am Mühlenberg 1, D-14476 Golm, GerMANY 